\title{
Polysèmes
}

Revue d'études intertextuelles et intermédiales

17 | 2017

L'art intempestif-La démesure du temps

\section{L'art du temps « extraordinaire »}

The Art of "Extraordinary" Time

\section{Camille Fallen}

\section{(2) OpenEdition}

Journals

Édition électronique

URL : http://journals.openedition.org/polysemes/1825

DOI : $10.4000 /$ polysemes. 1825

ISSN : 2496-4212

Éditeur

SAIT

\section{Référence électronique}

Camille Fallen, «L'art du temps « extraordinaire » », Polysèmes [En ligne], 17 | 2017, mis en ligne le 30 avril 2017, consulté le 09 mai 2019. URL : http://journals.openedition.org/polysemes/1825 ; DOI : 10.4000/polysemes.1825

Ce document a été généré automatiquement le 9 mai 2019.

Polysèmes 


\title{
L'art du temps « extraordinaire »
}

\author{
The Art of "Extraordinary" Time
}

\section{Camille Fallen}

Que l'art, parfois, soit dit intempestif suggère que l'art peut non seulement faire quelque chose du temps, mais aussi, au temps. Intempestif, l'art ne jouerait pas exclusivement sur la gamme de la formation ou de la transformation des formes. Simultanément, il toucherait au temps, à ses formes et à ses forces, soit à la plasticité ${ }^{1}$ du temps lui-même. Par conséquent, l'art serait aussi un art du temps, l'art de faire quelque chose du temps et au temps: l'art de produire un effet intempestif sur les formes internes ou externes, subjectives ou objectives, collectives ou historiques de la temporalité. La perception et la représentation de l'espace-temps seraient affectées par un art dit intempestif.

Or, ce qui était déplacé, inconvenant, importun, c'est-à-dire intempestif, ou bien ce qui restait encore à l'être, semble toujours finir par "faire époque» et par intégrer le dispositif général de l'Histoire de l'art et des arts : reconnu, absorbé et relevé comme sans reste dans la nomenclature des catégories artistiques, esthétiques et historiques connues, l'intempestif prend ainsi place dans un dispositif qui neutralise sa puissance de déplacer encore les catégorisations dans lesquelles il se trouve finalement enserré. Fournissant des critères d'intelligibilité normés, l'Histoire de l'art et des arts arraisonnerait les œuvres en les réifiant dans la temporalité figée de la connaissance historique. Comme le dénonce Nietzsche, cette domestication historique s'apparenterait alors à une forme de taxidermie muséologique. Ensevelissant l'intempestif sous les sédiments et les éloges funèbres d'une bienséance érudite, les institutions s'en feraient les fossoyeurs. Dans Considérations inactuelles, Nietzsche écrit : «Un phénomène historique, exactement et entièrement connu, totalement transformé en un phénomène cognitif, est pour celui qui le connaît un objet mort " (102). Une constellation de questions dont on ne sait si les lueurs ou les lucioles qui palpitent encore nous arrivent post mortem ou non accompagne dès lors notre notion : qu'est-ce qu'un objet d'art vivant? Qu'est-ce que garder l'œuvre d'art vivante, qu'est-ce que le temps vivant d'une œuvre et qu'est-ce qui, en elle, conserve la puissance d'échapper à sa réification historique ? Qu'est-ce qu'opérer à vif sur le temps et garder en vie la temporalité intempestive de l'art? Comment les puissances de dérangement de 
l'intempestif peuvent-elles survivre à l'Histoire? Comment l'intempestif peut-il paradoxalement " demeurer ", « durer ", " survivre » et rester à venir ? Selon quel ordre ou désordre du temps l'intempestif peut-il rester à contretemps? Selon quelle conjugaison impossible peut-il garder de l'événement en réserve, nous réserver encore de l'événement à venir ? Comment l'intempestif peut-il en venir à déranger l'Histoire de l'art et des arts une fois encore, plus d'une fois, longtemps après?

Reprenons. Cette façon de pointer le temps au titre de l'Histoire émane peut-être d'un certain aveuglement au temps, c'est-à-dire d'une certaine relève toujours à moitié réussie et à moitié manquée de ce qui reste intempestif, encore à venir, indéterminé et mouvant dans le temps lui-même. Nietzsche poursuit: "Car trop d'histoire ébranle et fait dégénérer la vie, et cette dégénérescence finit également par mettre en péril l'histoire elle-même » (103). Trop d'histoire ébranle la vie, le temps vivant, le temps survivant ou le temps à venir et à vivre encore. Faire revenir l'intempestif du passé, le voir venir au-delà de toute prévision comme depuis l'avenir aurait donc à voir, c'est notre première hypothèse, avec la plasticité temporelle des formes du temps vivant ou survivant, avec la vie et la survie spectrales, c'est-à-dire avec la temporalité spectrale des traces. Qui résiste en effet au temps, à la mort, au refoulement ou à l'oubli mieux que les spectres? En serait exemplairement témoin la Gradiva - ou " celle qui marche en avant » - dans Le Délire et les Rêves dans la Gradiva de W. Jensen ${ }^{2}$, Gradiva qui, pas à pas, nous conduit au dénouement intempestif de plusieurs temporalités entrelacées entre rêve, réalité, réminiscence, refoulement, histoires individuelles et Histoire.

Pas à pas, revenons donc nous aussi en arrière pour jouer tout d'abord de la forme contre le fond, ou encore d'une forme temporelle sans fond réel ni rationnel, sans fond ontologique ni logique: celle des traces spectrales. Que les arts et les artistes forment, inventent et informent leurs propres tables de conjugaison, qu'ils s'y entendent à conjuguer le temps autrement, que selon quelques-unes de ces tables anciennes ou nouvelles, l'art aille parfois à contre-courant du temps chronologique ou historique, jouant synchroniquement ou diachroniquement le contretemps, ce serait, nous dit l'Histoire de l'art, l'évidence. L'évidence que, depuis un certain temps, lui aussi historique, nous appelons l'intempestif : par exemple, l'intempestivité du pan de peinture renaissante analysé par Georges Didi-Huberman dans Devant le temps (la partie inférieure de la Madone des Ombres de Fra Angelico au couvent de San Marco) ${ }^{3}$. "Quand " l'art est intempestif, il a alors cette puissance de reconfiguration du passé, du présent et de l'avenir dont nous parle Georges Didi-Huberman, cette puissance de reconfiguration du temps et des temps qui nous saisit, par exemple, « devant une image ». Mais d'une autre façon, la proposition dont nous suivons ici la logique " "quand" l'art est intempestif... » est elle-même intempestive, c'est-à-dire déplacée ou mal scandée, ontologiquement ou temporellement : en elle réside déjà, comme recouvert, un temps ou le temps hors de ses gonds (« the time is out of joint " comme nous dit Hamlet). Car si «l'art est », là où il est question à la fois de l'Être et du temps, l'art ne peut pas «être» intempestif. Pour l'Être, intempestif - c'est-à-dire inconvenant, déplacé, importun, à contretemps -, est l'intempestif lui-même qui, strictement, n'Est pas, en tout cas, pas au sens de l'Être et du présent. Que l'Être soit au présent ce que l'intempestif n'Est pas annonce donc, pour l'intempestif, un autre temps que le temps dit « vulgaire » qui, selon Heidegger, est le temps tel qu'il a été pensé d'Aristote à Bergson ${ }^{4}$. Par conséquent, là où elle touche au temps, la question de l'art intempestif marque la différence entre deux temps, le temps vulgaire d'un côté et ce que d'un autre côté nous appellerons ici le temps 
" extraordinaire» ou anomal ${ }^{5}$, soit un temps qui s'excepte ou s'exempte du temps vulgaire : une temporalité autre qui ne se compte ni ne se raconte pas selon le temps ordinaire. Une temporalité autre qui ne fait pas l'Être mais le défait tel qu'il « est » et avec lui, défait le temps vulgaire, l'ordre du temps et des choses temporelles. Une temporalité quasi créatrice. Par exemple.

5 L'art intempestif ne peut " être » au sens du temps vulgaire, c'est-à-dire au sens de la présence, du présent et de la présentation. L'art intempestif ou l'art out of time, out of joint ne se présente plus comme l'un des rouages du dispositif perceptif, discursif, temporel et historique qu'il ne peut que détraquer. L'art intempestif entretient avec le temps et avec notre perception du temps une relation de détraquage de ses rouages, qu'ils soient sensibles, subjectifs, collectifs, rationnels, historiques ou tout à la fois. Il ne connaît pas l'ordre du temps, c'est-à-dire l'ordre de la préséance du présent, de la présence et de la présentation, pas plus sans doute qu'il ne connaît le commencement ou l'origine, le milieu ou le développement, la fin ou la finalité : il n'est pas déterministe, il n'est pas entéléchique, il n'est pas téléologique. Il n'est ni chronologique ni discursif. L'art intempestif n'obéit donc pas au temps mais le troue, le déchire, le décompose ou le recompose autrement: il fracture le continuum spatio-temporel pour donner lieu à un ordre (ou à un désordre) temporel qui ne lui préexistait pas. L'art intempestif, qui n'Est pas, présente donc l'imprésentable et l'impossible de l'Être et du temps. En un certain sens, notre problématique rejoint alors celle du contemporain qui, elle aussi, nécessite un discours quasi apophatique. Selon Agamben, le contemporain est en effet celui «qui a brisé les vertèbres de son temps (c'est-à-dire a perçu la faille ou le point de cassure), [qui] fait de cette fracture le lieu d'un rendez-vous et d'une rencontre entre les temps et les générations » (37-38). Brisure, cassure, césure, hétérogénéité du temps, voilà ce qui serait le signe tantôt du contemporain, tantôt de l'intempestif, le signe d'un temps autre, qui dans son présent même, ne se sera pas présenté, n'aura pas été vécu :

Le présent, poursuit Agamben, n'est rien d'autre que la part de non-vécu dans tout vécu, et ce qui empêche l'accès au présent est précisément la masse de ce que, pour une raison ou pour une autre (son caractère traumatique, sa trop grande proximité) nous n'avons pas réussi à vivre en lui. L'attention à ce non-vécu est la vie du contemporain. Et être contemporain signifie, en ce sens, revenir à un présent où nous n'avons jamais été. (35-36)

6 Mais tandis que le risque subsiste de prendre l'espace pour le temps, nous risquons ici de prendre les discours sur le temps pour le temps lui-même. Car reconfigurer le passé, le présent et l'avenir, c'est surtout reconfigurer l'ordre de nos discours sur l'histoire des temps, des périodes ou des époques de l'art. Par ailleurs, arriver à penser temporellement l'art intempestif, ce serait, d'une certaine façon, avoir résolu la question de savoir ce qu'est le temps, question qui, comme les questions métaphysiques, demeure irrésolue dans le sens où ni les philosophes, ni les physiciens ne s'entendent finalement sur la nature, l'existence ou l'inexistence du temps.

7 Poursuivons toutefois notre hypothèse comme s'il y avait du temps ou comme si le temps était. Qu'il y en ait ou qu'il soit, dans tous les cas, un point aveugle caractérise notre relation au temps, comme au temps qu'il fait pour le contemporain. Augustin écrivait ainsi au livre XI des Confessions: "Qu'est-ce donc que le temps? Si personne ne me le demande, je le sais; mais si on me le demande et que je veuille l'expliquer, je ne sais plus ». Pourtant, inventer son temps et sa temporalité, reprendre le temps, à zéro ou d'autre part, s'impose certainement à quiconque « crée ", à moins que ce ne soit le temps extraordinaire qui impose ou s'impose à la création. «Quand l'artiste se met au travail » 
écrit Andréï Tarkovski, "il doit croire qu'il est le premier à donner forme à un phénomène particulier, dans la manière même où il le ressent et où il le comprend" (131). Pour rester sur la piste des phénomènes artistiques, rappelons que pour Kant, le changement ne concerne pas le temps lui-même mais, justement, les phénomènes dans le temps. Par conséquent, ce n'est plus seulement la question d'un temps autre qui va nous intéresser ici mais celle, pour ainsi dire contiguë, du changement autre, c'est-à-dire du devenir autre et autrement que selon le devenir ordinaire (ou vulgaire) des phénomènes. Ce que, cette fois, nous appellerons devenir extraordinaire. L'art intempestif, facteur d'un devenir extraordinaire s'insérant dans le temps, le brisant ou le scandant de façon à faire quelque chose d'autre du temps et au temps, c'est ce que, d'un côté pour marquer l'hétérogénéité, de l'autre pour renvoyer à Michel Foucault, nous articulerons ici à la notion d'hétérochronie. Ce terme, que nous détournons ici, est employé par Michel Foucault dans Les Hétérotopies ${ }^{6}$ : «Il se trouve, écrit-il, que les hétérotopies sont liées le plus souvent à des découpages singuliers du temps. Elles sont parentes, si vous voulez, des hétérochronies » (30). Que penser alors des hétérochronies artistiques? Nous n'utilisons pas nécessairement ici ce terme pour dire les galeries, les musées et autres lieux d'exposition institutionnels ou non, mais pour désigner les objets de l'activité artistique en tant que celle-ci s'affaire à des transformations inédites du temps, à des opérations qui nous font sortir des perceptions, des représentations ou des conceptions ordinaires du temps. L'hétérochronie caractériserait alors les phénomènes artistiques qui, pesant autrement sur notre sens de la perception temporelle, nous affecteraient - le temps, le sens du temps, du changement et des phénomènes - autrement. Nous appellerons ainsi hétérochronies artistiques ou anomalies hétérochrones ces hétérotopies manifestant non pas le temps «vulgaire » et ses différentes modalités, mais le devenir extraordinaire, la manifestation d'un temps en crise, d'une crise du temps ou simplement, d'un temps ou d'une temporalité autre, hétérogène. À propos d'opérations temporelles, de transformations du temps et de devenir extraordinaire, écoutons Valéry :

Wagner, comme Rembrandt, savait attacher l'âme du patient à quelque partie éclatante et principale; et cependant qu'il l'enchaînait et l'entraînait à ce développement tout-puissant, il faisait naître dans l'ombre de l'ouie, dans les régions distraites et sans défense de l'âme sensitive, - des événements lointains et préparatoires, - des pressentiments, des attentes, des questions, des énigmes, des commencements indéfinissables... C'est là construire un art à plusieurs dimensions, ou organiser, en quelque sorte, les environs et les profondeurs des choses explicitement dites. Il me souvient d'un temps fort éloigné où je m'inquiétais si des effets analogues à ceux-ci pourraient se rechercher raisonnablement en littérature.

8 Lorsque la matière "plastique" - que celle-ci soit matérielle, virtuelle ou encore picturale, sonore, verbale - est modelée, créant des formes singulières et signées, d'autres formes temporelles sont produites ou se produisent, reconfigurant autour d'elles comme « devant l'image » nos sens du temps et de l'Histoire, c'est-à-dire aussi nos sens-ibles, à la croisée de la sensibilité et du sens. Ces temporalités, il convient de les analyser à chaque fois, singulièrement, selon la singularité des artistes, même lorsque, comme les physiciens, nous n'avons pas les outils conceptuels et techniques pour penser les temps des commencements et de la " création ». De la sorte, aujourd'hui même, tandis que nos existences, qu'il s'agisse de l'art ou "des temps qui courent ", sont enchaînées aux puissants motifs du marché, du spectacle, de l'accélération de la mondialisation et de la crise, il faudrait se demander ce qui, intempestif, peut encore se loger dans l'ombre de l'ouïe, dans l'ombre de la vue, dans l'ombre des sens-ibles et de leur espace-temps. Est-ce que l'art 
a encore suffisamment de dimensions, d'environs et de profondeurs pour nous garder, dans tous les sens du terme, des temps et d'autres temporalités à venir?

L'intempestif - et ce serait là son imprésentable et son impossible - aurait alors à se frayer une voie à travers la fermeture de l'Histoire, des histoires et du temps. Cette clôture de l'événement dans laquelle nous sommes parqués s'appelle aujourd'hui même capitalisme et mondialisation. Or, paradoxalement, cette clôture de l'événement, qui dessine un monde sans dehors où ne cessent de se produire et de se reproduire des événements sans événement, partage quelque chose de la structure de l'intempestif qui garde l'événement en réserve et ne se présente pas au présent. L'intempestif à venir dans le franchissement ou l'éclatement de cette clôture prendrait alors le risque d'être confondu avec la série des événements sans événement qui justement paraissent la clôturer. Ceux dont fait état Zizek et qui consistent à accueillir l'Autre une fois privé de son altérité :

On trouve aujourd'hui sur le marché de nombreux produits dont ont été éliminées les propriétés malignes : café sans caféine, crème sans matière grasse, bière sans alcool... Et la liste continue : pourquoi pas une partie de jambes en l'air virtuelle, une guerre sans guerre, comme Colin Powell l'a proposé dans sa doctrine de la guerre sans victimes (de notre côté, bien sûr) ? La politique sans politique, comme on la redéfinit actuellement en la réduisant à un art de l'expertise administrative? Et pourquoi pas, comme le conçoit aujourd'hui le multiculturalisme libéral et tolérant, l'expérience de l'Autre, mais privé de son Altérité (cet Autre idéalisé qui danse de façon fascinante, nourrit une approche écologique, saine et holiste de la réalité, dans lequel un phénomène comme celui des femmes battues n'a plus cours... ) ? (30-31)

10 Pour décrire ce type de phénomènes, Slavoj Zizek parle de «chocolat laxatif » ou de " croyance décaféinée ", soit de produits contenant leur propre négation. Des sentiments, des engagements, des événements faits de ce qui les vide de leur effectivité et de leur sens. Chocolat sans chocolat, étranger sans altérité, politique sans politique, c'est-à-dire pas de chocolat, pas de politique, pas d'étranger mais juste une chose sans chose s'effectuant sans raison ni cause, quasi ex nihilo et pour rien d'autre que le marché. Non plus « la rose sans pourquoi » d'Angelus Silesius mais le sans pourquoi sans rose qui vient s'ajouter à tous les «comme si cela n'avait pas eu lieu» de la vie même ${ }^{7}$. Or, là où intempestif et événement sont liés, il y a d'abord et souvent, rappelons-le, séparation, scission, exclusion, espacement et donc contretemps, diachronie, mais aussi solitude, isolement, esseulement, honte, refoulement et parfois, délire ou folie. Absence de récit dans le récit, anhistoricité, telles sont alors les traces sans traces de l'intempestif avec pour éventuelles figures le Bartleby de Melville, ou encore le personnage du père dans le Sacrifice de Tarkovski. L'un et l'autre incarnent le retrait, la dysharmonie et la butée du temps qui fait événement là même où l'événement n'est pas celui que l'on croit ou que l'on voit. Dans le Sacrifice, le père qui reste muet, brûle sa maison et passe pour fou aux yeux de tous, accomplissant ainsi le vœu par lequel, inaudible, invisible, hors du sens comme hors du temps, il sauve le monde. La fin du monde n'aura pas lieu, exceptée celle du père qui met ainsi fin au sien. Le vers de Paul Celan : Die Welt ist fort, ich muss dich tragen (Le monde s'en va, il faut que je te porte) exploré par Jacques Derrida dans Béliers ${ }^{8}$ exhibe dès lors son revers ou sa doublure : le monde reste, je dois porter sa perte ou bien, pour que le monde reste (le tien par exemple), je dois, invisible, inaudible, hors du sens et comme hors du temps, perdre le mien. De son côté, la portée du I would prefer not to de Bartleby à Wall Street résonne comme le retrait et le refus d'un monde qui gagne à se perdre. Dans les deux cas toutefois, si la notion d'événement emprunte au messianique, 
l'événement artistique intempestif n'est pas de l'ordre d'un spectacle où nous nous précipiterions tous ensemble en même temps. Comme si l'intempestif traçait les contours d'une kénose du temps, d'un temps manquant, absent parce qu'interrompu ou anéanti, ouvrant dès lors la blessure de l'irréversible à œuvrer pour qu'arrive l'impossible. Que l'on songe à Kafka dans « Un Médecin de campagne » : «Trompé ! Trompé ! Il suffit d'une fois : j'ai obéi à tort à la sonnette de nuit... C'est irréparable à jamais » (128). Ou encore, dans « Devant la loi » : «Ici nul autre que toi ne pouvait pénétrer, car cette entrée n'était faite que pour toi. Maintenant, je m'en vais et je ferme la porte» (138). Dans Ainsi parlait Zarathoustra, Nietzsche écrit: "Le vouloir ne peut rien sur ce qui est derrière lui. $\mathrm{Ne}$ pouvant détruire le temps ni l'avidité dévorante du temps, telle est la détresse la plus solitaire du vouloir» (187). Vouloir s'en prendre au temps du «fait accompli » ou au temps irréversible, irréparable ou perdu, c'est alors vouloir l'impossible et, d'un geste multiple, le rejouer d'une façon ou d'une autre pour le réajuster, le réparer, le transformer, le rédimer ou l'abolir. C'est aussi chasser ou rappeler les vivants et les morts, voir et faire venir l'avenir, catastrophique ou messianique. Autrement encore, c'est vouloir un éternel retour. Mais que le temps revienne deux fois le même, exactement, ou que l'on se baigne deux fois dans le même fleuve, Borges revient souvent sur cette question ${ }^{9}$ et ce serait la fin $d u$ temps. En ce sens, l'Idée de Beau, parce qu'elle fait entrevoir et désirer l'éternité, ce qui, le Même, se baigne dans tous les fleuves différents, donne un avant-goût de la fin du temps et peut-être, de la fin des temps : imitation d'imitation de l'Apocalypse. L'intempestif serait donc aussi l'histoire sans histoire du désir contrarié, de sa puissance déroutée, là où seuls survivent encore les spectres, en voie de résurrection ou non : désir qui déraille hors du temps quand le désir ne serait pas lui-même ce temps contrarié, anticipé mais différé. Le désir intempestif, artistique, révolutionnaire, messianique, là où les uns empruntent aux autres et vice versa, ce serait alors qu'il se passe quelque chose là où le désir ne passe pas. Qu'il se passe quelque chose contre le temps, dans sa case ou son laps vide, manquant ou déplacé, qu'un événement survienne « là », qui puisse renverser le temps et l'Histoire. Faire quelque chose au temps, au fait accompli ou inaccompli c'est ainsi ce que l'on croit parfois désirer le plus, comme Porc-Épic dans Stalker ${ }^{10}$.

\section{Post-scriptum}

11 Beaucoup de choses auront été dites et écrites sur les amoncellements de vêtements, manteaux, écharpes, jupes, pantalons, pull-overs de "Personnes», l'installation de Christian Boltanski au Grand Palais. Pourtant, d'une certaine façon, il semble que l'intempestif se soit monumentalement dissimulé sous nos yeux : car déni, occultation ou aveuglement, dans la masse de ces vêtements rassemblés qui, à travers les corps absents, représentent les êtres humains pour Boltanski, il n'y avait pas une jupe, pas un pantalon, pas une culotte, pas un caleçon, pas une chaussette, pas un soulier. Que des hauts et pas de bas. Et surtout, semble-t-il, personne pour le dire. Comme si ce fait devait être indifférent, insignifiant ou invisible. Si bien que dans cet amoncellement de vêtements rappelant la Shoah, le cimetière ou les exécutions massives (avec la grue évoquant le doigt de Dieu, du destin ou du hasard qui arrache à la vie), cette absence et ce silence devenaient aussi bruyants que les battements des cœurs enregistrés qui résonnaient dans le Grand Palais. Déraillement du temps, des histoires et des raisons de la chose artistique, quelque chose s'effectuait donc là sans cause, quasi ex nihilo, à vide mais par son absence, 
créait le contretemps hors de tous les temps suggérés. Ce jugement dernier sans culotte et sans pantalon abritait ironiquement l'œil sonore d'un cyclone qui, hors du temps commun, semblait regarder le monde courir à l'événement ou à la catastrophe comme à l'aveuglement. Même si Boltanski est familier de ces installations à moitié vêtues, quelques critiques et spectateurs s'inquiétèrent de ce que l'on allait faire de ces tonnes de vêtements, manteaux, écharpes, pull-overs, jupes, pantalons. Comme si l'artiste avait parié : ils ne verront pas. Ou encore : ils ne diront rien. Intention délibérée ou non, ce qui importe là, c'est la structure intempestive de cet événement sans événement, la structure de ce que l'on tait, de ce que l'on ne voit pas et/ou de que l'on ne dit pas là où il y a gêne, honte, refoulement : la structure du déni silencieux qui, de tout temps, entraîne les uns ou les autres à la mort, dans les camps ou ailleurs. Au cœur même d'un temps sans reconfiguration possible.

En ce sens, la fabrique de l'événement artistique public mime l'intempestif qui, lui, n'a certainement lieu qu'à ne pas avoir lieu ou bien ailleurs, autrement, à contretemps. Intempestif est alors le nom de ce qui demeure séparé, exclu ou refoulé, fût-ce de l'événement le plus visible et le plus spectaculaire. Il a beau s'étaler sous nos yeux, nous restons aveugles par le fait même de croire voir et savoir exactement ce dont il s'agit là, en termes d'art, de temps et d'Histoire. Comme si l'Institution rhabillait automatiquement l'intempestif pour éviter honte, gêne, impudeur, personnelle ou collective, comme si, machinalement, l'intempestif était recouvert ou chassé et avec lui, peut-être, la nudité rédimée de tout événement messianique.

Que l'intempestif puisse nous garder des temps à venir, dans tous les sens de cette expression, ce serait peut-être, pour reprendre le titre de Borges, faire de l'art du temps un Jardin aux sentiers qui bifurquent. Non seulement dans l'espace mais aussi dans le(s) temps. Un art dont on puisse dire, comme dans la nouvelle : «Je laisse aux nombreux avenirs (non à tous) mon jardin aux sentiers qui bifurquent» (99). Mais puisque l'intempestif n'Est pas, puisqu'il ne se présente pas au présent, puisqu'il est donc ici partout manquant, puisque, donc, l'intempestif n'aura pas lieu hic et nunc, je vous laisse abruptement dans ce jardin.

\section{BIBLIOGRAPHIE}

Agamben, Giorgio. Qu'est-ce que le contemporain ? (2005). Trad. Maxime Rovere. Paris : Payot \& Rivages, 2008.

Borges, Jorge Luis. Enquêtes (1957) suivi de Entretiens. Trad. Paul et Sylvia Bénichou. Paris : Gallimard, « Folio Essais », 1992.

Borges, Jorge Luis. « Le Jardin aux sentiers qui bifurquent » (1941). Fictions. Trad. Roger Caillois. Paris : Gallimard, « Folio », 1991.

Derrida, Jacques. Béliers Dialogue interrompu : entre deux infinis, le poème. Paris : Galilée, 2003.

Kafka, Franz. « Un Médecin de campagne » (1920). La Métamorphose. Paris : Gallimard, « Folio », 1955. 
Kafka, Franz. « Devant la loi » (1915). La Métamorphose. Paris : Gallimard, « Folio », 1955.

Didi-Huberman, Georges. Devant le temps, histoire de l'art et anachronisme des images. Paris : Éditions de Minuit, 2000.

Fallen, Camille. L'Anomalie créatrice. Préface de Jean-Luc Nancy. Paris : Kimé, « La philosophie en cours », 2012.

Foucault, Michel. Les Hétérotopies (1984). Le Corps utopique, les hétérotopies. Paris : Lignes, 2009.

Freud, Sigmund. Le Délire et les Rêves dans la Gradiva de W. Jensen (1907). Trad. Paule Arbex et RoseMarie Zeitlin. Paris : Gallimard, « Folio Essais », 1996.

Heidegger, Martin. Être et temps (1927). Trad. François Vezin. Paris : Gallimard, « Bibliothèque de Philosophie », 1990.

Malabou, Catherine. L'Avenir de Hegel. Plasticité, temporalité, dialectique. Paris : Vrin, « Bibliothèque d'Histoire de la Philosophie ", 1996.

Melville, Herman. Bartleby le scribe (1853). Trad. Pierre Leyris. Paris : Gallimard, « Folio », 1996.

Negri, Antonio. Art et multitude, neuf lettres sur l'art. Trad. Judith Revel, Nicolas Guilhot, Xavier Leconte et Nicole Sels. Paris : Fayard, « Mille et une nuits », 2009.

Nietzsche, Friedrich. Considérations inactuelles I et II (1873-1876). Paris : Gallimard, « Folio Essais », 2007.

Nietzsche, Friedrich. Ainsi parlait Zarathoustra (1883-1885). Trad. Geneviève Bianquis. Paris : Flammarion, « GF », 1996.

Zizek, Slavoj. Bienvenue dans le désert du réel (2002). Trad. François Théron. Paris : Flammarion, «Flammarion Documents et essais », 2005.

Tarkovski, Andreï. Le Temps scellé (1986). Trad. Anne Kichilov et Charles H. de Brantes. Paris : Cahiers du Cinéma, « Petite Bibliothèque des Cahiers du cinéma », 2004.

Valéry, Paul. Le Retour de Hollande (1926). Variété, Études Philosophiques in CEuvres I, Paris :

Gallimard, « Pléiade », Paris, 1957.

\section{NOTES}

1. Le terme "plasticité » est un terme dont nous devons l'élaboration philosophique à Catherine Malabou, notamment dans son ouvrage L'Avenir de Hegel. Plasticité, temporalité, dialectique, Paris : Vrin, 1996.

2. Sigmund Freud, Le Délire et les Rêves dans la Gradiva de W. Jensen, traduit de l'allemand par Paule Arbex et Rose-Marie Zeitlin, Gallimard, « Folio Essais », Paris, 1996.

3. Georges Didi-Huberman, Devant le temps, Paris : Éditions de Minuit, 2000.

4. Jean-Luc Nancy nous a rappelé que Derrida, dans Ousia et Grammè, posait la question de savoir si un autre temps que le temps qualifié de "vulgaire» par Heidegger pouvait exister. Nous aimerions plus tard consacrer le temps nécessaire à cette question.

5. Je me permets de noter ici que ce concept a été élaboré dans notre thèse de doctorat Pour penser l'anomalie et qu'il a donné lieu à un premier ouvrage L'Anomalie créatrice, préface de JeanLuc Nancy, Kimé, Paris, 2012.

6. Michel Foucault écrit: «Eh bien! Je rêve d'une science - je dis bien une science - qui aurait pour objet ces espaces différents, ces autres lieux, ces contestations mythiques et réelles de l'espace où nous vivons. Cette science étudierait non pas les utopies, puisqu'il faut bien réserver 
ce nom à ce qui n'a vraiment aucun lieu, mais les hétéro-topies, les espaces absolument autres ; et forcément, la science en question s'appellerait, s'appellera, elle s'appelle déjà "l'hétérotopologie" " (25).

7. «L'impuissance n'est pas la nullité de l'effet de quelque chose qui se voulait puissant, mais la qualité primaire, radicale, de la production. Le marché, son pouvoir ont absorbé toutes les puissances pour leur ôter la possibilité de devenir singulières, de valoir pour quelqu'un ou quelque chose. De produire. La créativité est retirée. L'impuissance est la matière même du discourir, du communiquer, du faire. Non pas le néant, mais l'impuissance. La grande machine circulatoire du marché produit le rien de la subjectivité. Le marché détruit la créativité. La puissance est retirée » (Negri 36-37).

8. Jacques Derrida, Béliers Dialogue interrompu : entre deux infinis, le poème, Paris : Galilée, 2003.

9. «Ne suffit-il pas de la répétition d'un seul terme pour disloquer et confondre la série entière du temps?» (Borges 1992, 233).

10. «Je rappelle que lorsque les héros du film commencent leur voyage vers la Zone, leur but est d'atteindre une chambre où, leur dit-on, leurs vœux les plus secrets seront exaucés. Alors que l'Écrivain et le Savant sont guidés par le Stalker, et qu'ils franchissent les espaces étranges de la Zone, celui-ci leur raconte à un moment donné l'histoire vraie ou légendaire d'un autre Stalker, surnommé Porc-Épic, qui s'était rendu dans ce lieu secret pour demander le retour à la vie de son frère, mort par sa faute. Mais de retour chez lui après avoir visité la chambre, Porc-Épic avait découvert qu'il était devenu fabuleusement riche. La Zone avait en fait réalisé son vœu le plus secret, et non pas celui dont il voulait ou essayait de s'inspirer. Et Porc-Épic était allé se pendre » (Tarkovski 228-229).

\section{RÉSUMÉS}

Cet article s'intéresse à la possibilité d'une temporalité artistique intempestive, plastique, c'est-àdire à un temps extraordinaire différant du temps ordinaire et qui garderait vive ou vivante la capacité de l'art à faire évènement, à reconfigurer le temps ou à franchir la clôture de l'Histoire. Cette temporalité qui garde en réserve de l'événement ou cet événement qui, hors des catégories ontologiques traditionnelles, garde du temps en réserve, est ici placé sous le signe de la transformation intempestive des formes, du devenir extraordinaire et des hétérochronies ou anomalies hétérochrones. Par opposition ou différenciation avec les schèmes ordinaires de la représentation du temps, il s'agit de saisir ce que l'art fait à notre sens de la temporalité en contrepoint de ce que l'Histoire fait à l'art.

This article deals with the possibility of an untimely artistic temporality, that is to say of an extraordinary time differing from ordinary time, which would keep alive the capacity of art to become an event, to re-configure time or to reach beyond the limits of History. This temporality that keeps some event in reserve or this event which keeps time in reserve (outside traditional ontological categories) is here under the sign of the untimely transformation of forms, extraordinary becoming and heterochronies or heterochronous anomalies. In opposition with the ordinary schemes of our representation of time, it is a matter of seizing what art does to our sense of temporality as opposed to what History does to art. 


\section{INDEX}

Mots-clés : art, intempestif, temps ordinaire, devenir, anomalies hétérochrones

Keywords : art, untimely, ordinary time, becoming, heterochronous anomalies

\section{AUTEUR}

\section{CAMILLE FALLEN}

Camille Fallen est chercheur associé à l'Université Paris 8, laboratoire L.L.C.P. Elle a été membre permanent du Collège des études juives et de philosophie contemporaine - Centre Emmanuel Lévinas, Université Paris-Sorbonne (2012-2016). Docteur en philosophie, professeur bi-admissible de philosophie, qualifiée aux fonctions de MCF (CNU 17), elle est rédactrice de la revue De (s)générations http://www.desgenerations.com. Son dernier ouvrage publié s'intitule L'Anomalie créatrice (préface de Jean-Luc Nancy, Paris : Kimé, 2012). Elle travaille actuellement à la rédaction de Esthétiques anomales (à paraître aux éditions Kimé). Elle a co-édité l'ouvrage collectif intitulé Poétiques de Michel Deguy (dirigé par Pascal David, Camille Fallen, Bénédicte Gorrillot, Elisabeth Rigal, Martin Rueff, actes réunis de la soirée "Michel Deguy" à la Maison de l'Amérique latine, mai 2013), et les actes du colloque de La Tourette, octobre 2016 (à paraître fin 2018 dans Revue des Sciences Humaines). Activités détaillées de recherches : http://blog.camille-fallen. 